\title{
Metabolic enzymes link morphine withdrawal with metabolic disorder
}

\author{
Xi Jiang ${ }^{1}$, Jing $\mathrm{Li}^{2}$, Lan $\mathrm{Ma}^{1}$ \\ ${ }^{I}$ National Laboratory of Medical Neurobiology and Pharmacology Research Center, Shanghai Medical College and Institutes of \\ Brain Science, Fudan University, Shanghai 200032, China; ${ }^{2}$ Mental Health Center, Huaxi Hospital, Sichuan University, Chengdu \\ 610041,China.lanma@fudan.edu.cn
}

Cell Research (2007) 17:741-743. doi: 10.1038/cr.2007.75; published online 13 September 2007

Energy metabolism is a fundamental biological process that is vital for the survival of all species. Disorders in the metabolic system result in deficiency or redundancy of certain nutrients, including carbohydrates, lipids, amino acids, etc. Abnormality of the energy metabolism system leads to a number of metabolic diseases, such as the metabolic syndrome. Broadly speaking, the term "metabolic diseases" now tends to be widened to the category that refers to all diseases with metabolism disorder. It is shown that many diseases associate with metabolic disorders. For example, most malignant tumors progress with mal-nutrition and high consumption, that is, cachexia. Many components of the energy metabolism system, such as lactate dehydrogenase (LDH), are now widely applied in clinical examinations as special markers for tumors and some other diseases. Opioid dependence and addiction are neurobiological diseases associated with malregulation of the metabolic system. However, how chronic drug administration induces metabolic abnormality is not understood. In a recent issue of Cell Research, research group of Jing-Gen Liu [1] reports an interesting discovery that three metabolic enzymes are changed in mice after chronic morphine treatment, suggesting new roles of metabolic enzymes as a potential link that associates metabolic disorder with opioid dependence.

The process of energy metabolism is very complex, a major part of which is the tricarboxylic acid cycle. The tricarboxylic acid cycle contains a variety of different enzymes and substrates, such as the pyruvate dehydrogenase (PDH). Functioning as a complex, PDH oxidizes pyruvate, by converting it into acetyl-CoA, and produces the bulk of ATP. Both the pyruvate cycle and PDH itself are under stringent regulation. PDH activity is regulated by its phosphorylation state, being most active in the deposphorylated state. PDH dysregulation may lead to metabolic diseases. Lack of PDH activity has long been recognized as the most common cause of primary lactic acidosis in infancy. PDH deficiency occurs in rats after exposure to high glucose or free fatty acid, indicating its close relationship with diabetes. It has been proposed recently that PDH kinase inhibitors might be an effective therapy for Type II diabetes [2]. On the other hand, PDH increment has been reported in hypertrophic cardiomyocytes induced by hypoxia-reoxygenation. Kobayashi et al. reported that high level of cardiac work increases PDH activity, which associates closely with reduced mitochondrial NADH/NAD ${ }^{+}$ratios and acetyl CoA/CoA ratios [3].

Accumulating evidence stresses that dysregulation of energy metabolism system relates with cerebral diseases or psychiatric disorders. It has been demonstrated that ischemic brain injury results in the reduction of energy metabolism. Loss of PDH activity and reduced expression level were found in vulnerable neurons after cerebral ischemia and reperfusion, which may result in the reduced cerebral glucose and oxygen consumption [4-5]. Studies also link metabolic abnormality to chronic cerebral diseases and dementia, such as Alzheimer disease (AD). Metabolic syndrome has been recently reported to associate with increased risk for $\mathrm{AD}$. Cognitive abnormalities of $\mathrm{AD}$ to a large extent result from decreasing brain metabolism. Mitochondrial dehydrogenases affecting energy transfer are frequently altered in $\mathrm{AD}$. One of the $\mathrm{AD}$ associating protein, the amyloid precursor protein (APP) can target mitochondria, and reduce cytochrome oxidase activity and ATP level [6]. It has been reported that mitochondrial movement is impaired in neurons of AD patients [7], which correlates with altered expression of APP. 
Drug dependence and addiction are chronic, relapsing brain disorders, manifesting as brain changes resulted from chronic drug exposure. Addiction to drugs, such as alcohol, has been well shown to be related with abnormality of the energy metabolic system. Development of opioid dependence involves a variety of different signaling pathways and neurotransmitters, among which glutamate and GABA play crucial roles. It has been shown that ATP could inhibit glutamate synaptic release in the hippocampus, suggesting its importance in modulating neurotransmitters and glutamate signal transduction [8]. In the brain of opioid addicts and opiate dependent animals, metabolic processes such as glycolysis and the tricarboxylic acid cycle are reduced [9-10]. Moreover, glucose has been shown to suppress acute morphine withdrawal signs, attenuating acute morphineinduced memory impairment and increment of locomotor activity [11]. These studies suggest that the energy metabolic system plays an important part in opioid dependence. Therefore should we treat opioid dependence as one new type of energy metabolism disorder? If so, what is the molecular mechanism for such kind of dysregulation?

The paper by Chen et al. [1] shows for the first time, through 2-dimensional gel electrophoresis and mass spectrometry, that three key components of the energy metabolism system are regulated by opioid. In hippocampus of chronic morphine-treated mouse, the expression levels of $\mathrm{E} 2$ component of the pyruvate dehydrogenase complex (PDHC-E2), lactate dehydrogenase 2 (LDH2), and Fe-S protein 1 of NADH dehydrogenase are decreased, accompanied with reduced ATP production and impaired glucose metabolism. Their finding directly links chronic morphine treatment to energy metabolism disorder. Furthermore, they observe that both intrahippocampal and intraperitoneal injection of D-glucose could suppress naloxone-precipitated morphine withdrawal jumping and memory impairment in acute morphine-treated mice, indicating a potential metabolic treatment for morphine withdrawal symptoms.

The results of Chen et al. disclose a possible mechanism of morphine dependence and morphine treatment-induced memory impairment. Their study suggests that chronic morphine treatment may down-regulate certain components of the energy metabolism system, inhibit glucose metabolism and ATP production, and thus disturb the releasing of neurotransmitters, such as glutamate, to intrigue withdrawal syndrome and memory impairment (Figure 1).

This work raises an important question: why are there differences in acute and chronic morphine-treatment? As proposed by the authors, metabolic enzymes do not change in acute morphine-treated mouse hippocampus, and glucose can only relieve withdrawal syndrome and memory impairment in acute treated animals. Does this mean that hypoglycemia is one of the causes of acute morphine-treatment induced withdrawal syndrome? Acute morphine treatment may result in reversible glucose-ATP transition abnormality and chronic treatment may lead to irreversible reduction of enzymes, but why? More experiments are required to address these questions.

Moreover, the way through which morphine treatment alters energy metabolism and how energy metabolism affects withdrawal are still enigmatic. It has been reported by Li et al. that cholinergic system is involved in reversal of morphine withdrawal-induced memory impairment in

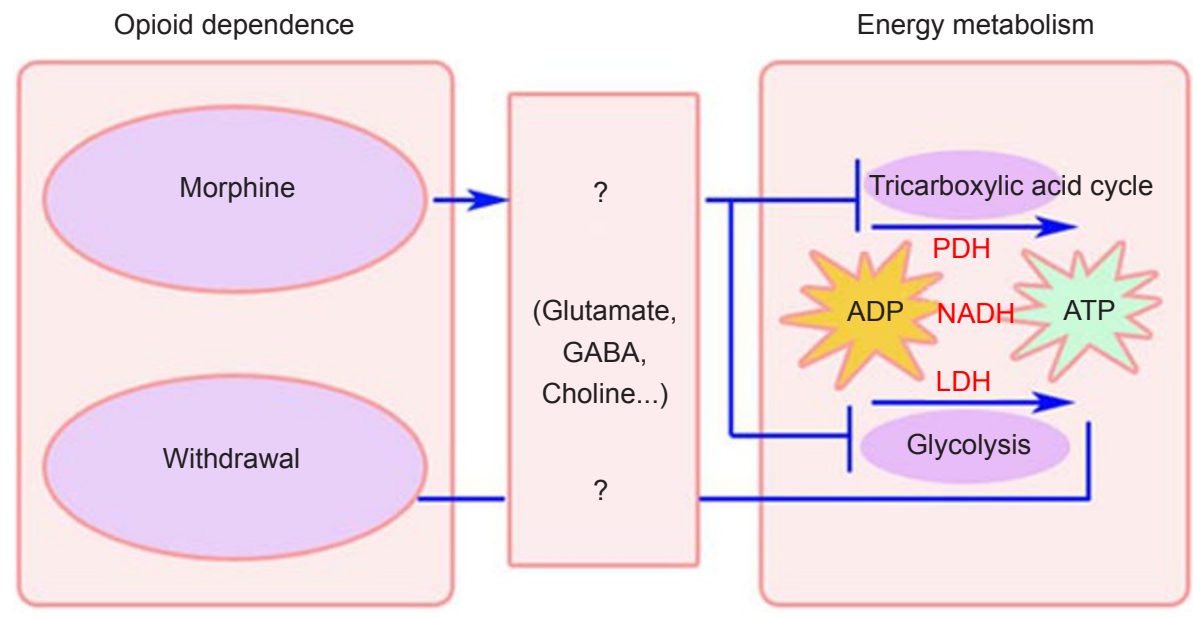

Figure 1 Model for the role of energy metabolism enzymes in morphine dependence. Morphine treatment can down-regulate the expression level of certain metabolic enzymes, including PDH, LDH, and NADH, and thus impairs the energy metabolism. The reduction of ATP is associated with morphine withdrawal symptoms and impairment in memory. 
mice [12]. Then what signal transduction results in the change of the glucose level and ATP production? How can we explain the alterations in metabolic enzymes following chronic morphine treatment? These questions need to be further investigated.

The work by Chen et al. proposes a new model linking opioid dependence and metabolic system. In such a model, opioid treatment plays a role in regulating energy metabolism and the latter in turn influences withdrawal symptoms. More studies should be done to confirm the regulations of the metabolic enzymes in morphine dependence, learning and memory. This would help elucidate the precise molecular mechanisms for the physical and psychiatric disorders caused by morphine treatment. If we can find out the way the metabolic enzymes are regulated and the way they regulate morphine withdrawal symptoms, we may explain morphine dependence in a new way.

\section{Acknowledgments}

The work in authors' laboratory was supported partially by grants from the Ministry of Science and Technology of China (2003CB515405 and 2005CB522406).

\section{References}

1 Chen XL, Lu G, Gong YX, et al. Expression changes of hippocampal energy metabolism enzymes contribute to behavioral abnormalities during chronic morphine treatment. Cell Res 2007; 17:689-700.

2 Mayers RM, Leighton B, Kilgour E. PDH kinase inhibitors: a novel therapy for Type II diabetes? Biochem Soc Trans 2005;
33:367-370.

3 Kobayashi K, Neely JR. Mechanism of pyruvate dehydrogenase activation by increased cardiac work. J Mol Cell Cardiol 1983; 15:369-382.

4 Bartnik BL, Hovda DA, Lee PW. Glucose metabolism after traumatic brain injury: estimation of pyruvate carboxylase and pyruvate dehydrogenase flux by mass isotopomer analysis. J Neurotrauma 2007; 24:181-194.

5 Martin E, Rosenthal RE, Fiskum G. Pyruvate dehydrogenase complex: metabolic link to ischemic brain injury and target of wxidative stress. J Neurosci Res 2005; 79:240-247.

6 Holmquist L, Stuchbury G, Berbaum K, et al. Lipoic acid as a novel treatment for Alzheimer's disease and related dementias. Pharmacol Ther 2007; 113:154-164

7 Trimmer PA, Borland MK. Differentiated Alzheimer's disease transmitochondrial cybrid cell lines exhibit reduced organelle movement. Antioxid Redox Signal 2005; 7:1101-1109

8 Mendoza-Fernandez V, Andrew RD, Barajas-Lopez C. ATP inhibits glutamate synaptic release by acting at $\mathrm{P} 2 \mathrm{Y}$ receptors in pyramidal neurons of hippocampal slices. J Pharmacol Exp Ther 2000; 293:172-179.

9 Dodge PW, Takemori AE. Effects of morphine, nalnorphine and pentobarbital alone and combination on cerebral glycolytic substrates and cofactors of rats in vivo. Biochem Pharmacol 1972; 21:287-294.

10 Sherman AD, Mitchell CL. Effects of morphine and pain on brain intermediary metabolism. Neuropharmacology 1972; 11:871877.

11 Akunne HC, Soliman KF. Hyperglycemic suppression of morphine withdrawal signs in the rat. Psychopharmacol 1988; 96: $1-6$

12 Li Z, Wu CF, Pei G, Xu NJ. Reversal of morphine-induced memory impairment in mice by withdrawal in Morris water maze: possible involvement of cholinergic system. Pharmacol Biochem Behav 2001; 68:507-513. 